\title{
Substance-Induced Sexual Dysfunction
}

National Cancer Institute

\section{Source}

National Cancer Institute. Substance-Induced Sexual Dysfunction. NCI Thesaurus. Code C94350.

A disorder characterized by clinically significant sexual dysfunction (including impaired desire, impaired arousal, impaired orgasm, or sexual pain) that is fully explained by substance use. 